\title{
Experimental test of the tool for the external EMF removing dents on a car body
}

\author{
Yuriy Victor Batygin \\ Chief of the Physics Department, Kharkov National Automobile \& Highway University, Kharkov, Ukraine \\ Email address: \\ batygin48@mail.ru, y.batygin@yandex.ru \\ To cite this article: \\ Yuriy Victor Batygin. Experimental Test of the Tool for the External EMF Removing Dents on a Car Body. International Journal of \\ Energy and Power Engineering. Vol. 3, No. 4, 2014, pp. 204-208. doi: 10.11648/j.ijepe.20140304.14
}

\begin{abstract}
This paper is dedicated to experimental study of pulsed electromagnetic attraction based upon recently developed tool design - "Inductor System with an Attracting Screen (ISAS)" for the repair technology of the external dent removing on a car body. The concept of attraction in this inductor system is based upon induced currents flowing in the same directions in the accessory screen and in the sheet metal blank, which, according to Ampere law, results in attraction forces between the screen and sheet metal blank. The more detailed information about ISAS is represented by Batygin (2006 [7], 2014 [8]). The main feature of this system is that the inductor is placed on the external side of the accessory screen. So, the induced currents in the parallel flat screen and sheet blanks are being excited by the penetrating magnetic field of the external source. Should mark this tool is capable of applying attraction forces to sheet metals of any physical nature as magnetic and stainless steel or aluminum. The conducted tests have shown a high effectiveness of suggested tool for the non-contact external flattening a car body in real production conditions. The represented work was done in the Laboratory of the Electromagnetic Technologies of the Kharkov National Automobile \& Highway University, Ukraine.
\end{abstract}

Keywords: Electromagnetic Forming, Electromagnetic Attractive Force, Repair Technologies, Dent Removing

\section{Introduction}

The interest to the magnetic pulse attraction of thinwalled conductors has a long-standing history. Some different suggestions allowing artificial transforming the natural magnetic pressure in the magnetic attraction had appeared during first testing some field technologies. The practical side of the marked interest is initiated by the growing demand upon production operations by the effective restoration of the airplane and vehicle bodies with the dents. The process has to be realized from a body external side. It has to be flattening without disassembling and without damaging existing protective cover. Precisely Electromagnetic Forming (EMF) technologies satisfy to all listed requirements. The objective of this paper is representing some experimental results for a new manufacturing process of the sheet metal attraction through the recently developed experimental system. Its the main feature is what a source of the pulse magnetic field (inductor) is positioned behind the additional accessory screen. This process is one of the few suggestions generating attracting forces with much higher capabilities than vacuum forming, for example.

\section{Literature Review and Problem Formulation}

Historically and chronologically, USA can be considered as "pioneer" and a leader in a field of the technical system creation for the external removing dents in the sheet conductors. Trustworthiness of this fact is confirmed by Web-Sites of the well known Americans companies "ELECTROIMPACT"[1] and "FLUXTRONIC"[2], for example. They have been representing some EMF equipment for the exterior restoration of aircrafts wings and fuselage when access from the inside of the panel is very problematic if not impossible.

Physical essence of their suggestions consists in generating two frequencies of discharge: slow and fast. The fast discharge eliminates the slow field from the side of the blank facing to the coil while the slow field diffused through the thickness of the sheet metal deforms the blank. This physical principle was incorporated in a number of coil designs and configurations: the leading engineers 
suggested a method and apparatus for electromagnetically removing dents from conductive materials by introducing a slow discharge through a multiturn coil and a rapid pulse of countercurrent; they further developed the coil system by introducing a flux concentrator which is also known in the literature as a field shaper. They introduced a system of two coils and portable pulse generator generating both repelling and pulling forces to correct both concave and convex areas of the dent.

The European leader in area of the creation of the modern repair equipment is the well known concern "BETAG INNOVATION"[3]. The Web-Site of the concern contains many suggestions of the different modern technologies for flattening a car body. Among them there is so named "Magnetic Dent Remover" claimed by Meichtry R., Kouba I. (2008 [4]). This device permits eliminating dents by attraction of the designated parts of magnetic steel blanks only. Non-magnetic metals can not be restored. But undoubted advantage of "Magnetic Dent Remover" is a possibility of restoration from the external side without disassembling of the components and without damaging of existing paint and protective coating. For justice sake should mark that the action principle of this device was taken from Batygin et al. (2004 [5]). The physical essence of mentioned action principle was justified theoretically and experimentally rather later. As it was shown by Batygin et al. (2013 [6]) when the working frequencies of the acting field are low enough the attracting forces are prevailing over the repelling forces which are well known forces of the magnetic pressure. The attraction is caused by the magnetic properties of the sheet billet metal. The nature of repulsion is caused by the Lorentz forces.

The main numerical estimates have shown the next.

- For low frequency about $\sim 2 \mathrm{\kappa Hz}$ the integral action (in time!) of the magnetic forces on the ferromagnetic thin sheet metal blank is essentially prevailing over Lorentz forces. The sheet metal blank is being attracted to the inductor.

- When increasing frequency up to $\sim 8 \mathrm{kHz}$, in comparison with $\sim 2 \mathrm{kHz}$ the Lorentz-forces are growing more than factor of three while the magnetic forces are remaining unchanged. The integral action of the Lorentz-forces becomes prevailing, while the attraction forces are getting rather small: the electromagnetic repelling pressure on the sheet metal blank takes place.

A new concept of electromagnetic attraction based upon force interaction between unidirectional currents (the well known Ampere Law!) in the blank and in the accessory screen was formulated for the first time by Batygin et al. (2006 [7]). Physical essence of the idea and its first technical implementation, named as "Inductor System with an Attracting Screen (ISAS)", were described later (2014 [8]).

The first construction of the attraction tool consisted of the single turn inductor positioned between parallel plates of the screen and sheet metal blank which requires treatment by attracting forces. Identically directed eddy currents are induced in the metal of the screen and the sheet metal blank by the current flowing through the single turn coil. The proposed design requires rather low frequency of discharge to accomplish intensive penetration of the electromagnetic field through the thickness of the blank and to avoid repelling Lorentz forces, which are the natural result of interaction of magnetic field with a conducting environment.

This construction of the electromagnetic attraction tool has an essential demerit: the exciting inductor is located between screen and work-piece. In connection with this, a strength of the system is falling down and the Lorentz repulsion is rather strong due to the proximity of the inductor and the sheet work-piece metal.

The further development of the theory and practice of the EMF attracting tools based on the Ampere Law have led to new ISAS - construction suggested by Batygin et al (2013 [9]) where the exciting inductor is moved out of the working zone and positioned on the external side of attracting screen. The new ISAS consists of parallel flat circle inductor, accessory screen and sheet metal blank. The induced whirl-wind currents in the screen and blank metals are being excited by penetrated pulse magnetic field being generated by the external source.

This research paper intends to represent the experimental test of the "Inductor System with an Attracting Screen (ISAS)", as the tool for the external EMF flattening a car body with the dent when the magnetic field source - the circle flat inductor located outside of the accessory screen. In conclusion should mark that the detailed review of literature with analysis of the problem is given in the papers $[6,8]$.

\section{The Simulated Production Process}

The manufacturing operation by the external non-contact removing the dents on the car metal covering was chosen for the experimental test of the "Inductor System with an Attracting Screen (ISAS)" with external circle inductor. The given choice was initiated by the information on WebSite of the leading European concern "BETAG INNOVATION" [3] where was marked the actuality of this production process for the advanced technologies of the modern auto transport repair. A principle scheme of the experimental modeling the chosen production process is represented on Fig.1. It includes a sketch of the ISAS construction used in the test.

Should mark some features of the conducted experiment.

1. The outside straightening was being realized precisely from side of concavity.

2. The excitation of ISAS was realized by the outside source of the magnetic field. The circle inductor was positioned on the external side of the accessory screen. 


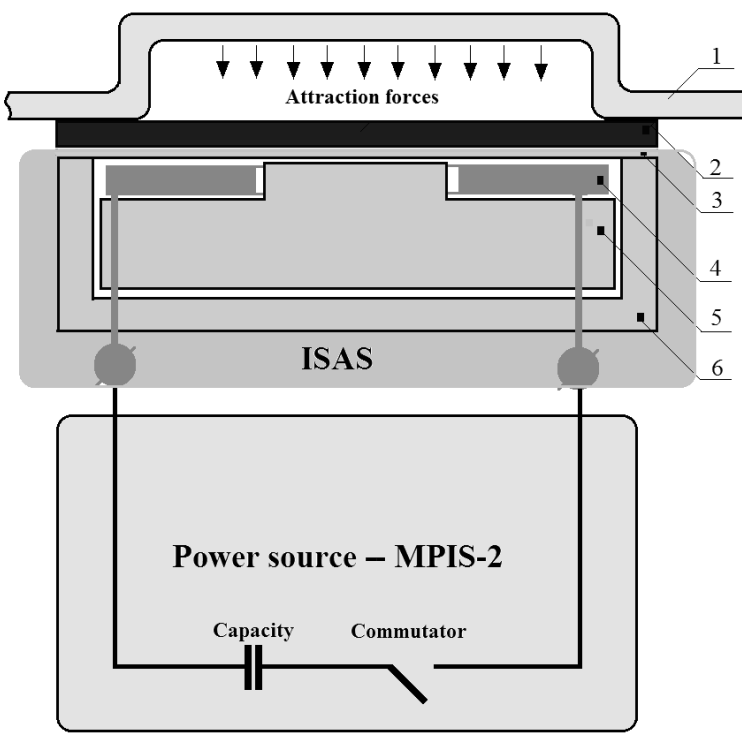

Fig 1. The principle scheme of the dent removing with help of the "Inductor System with an Attracting Screen (ISAS)", 1 - is the sheet metal blank with a dent, 2 - is the accessory attracting screen, 3 - is a dielectric insert, 4 - is the external exciting inductor, 5 - is a dielectric compaction, 6 -is the dielectric body; Power Source - is MPIS-2 ("Magnetic Pulse Installation Series" with $2 \mathrm{~kJ}$ energy stored):Capacity - is a capacitor bank; Commutator - is a block of thyristor switches.

\section{Equipment and Experimental Samples}

The draft of the ISAS experimental construction is represented on Fig.1 (see a part named ISAS). Its main components are the exciting circle inductor and the accessory attracting screen positioned in parallel.

Should mark the next very important circumstance. Speaking about the action result, the proposed ISAS is similar to the well-known classic electromagnet. But unlike the last one this tool permits to realize attraction not only ferromagnetics but metals of any physical nature as well. The inductor of ISAS was connected to the power source. It was MPIS-2 ("Magnetic Pulse Installation Series" with 2kJ stored energy) created in the Laboratory of the Electromagnetic Technologies and described in detail by Batygin et al (2013 [6], 2014 [8], 2014 [10]).

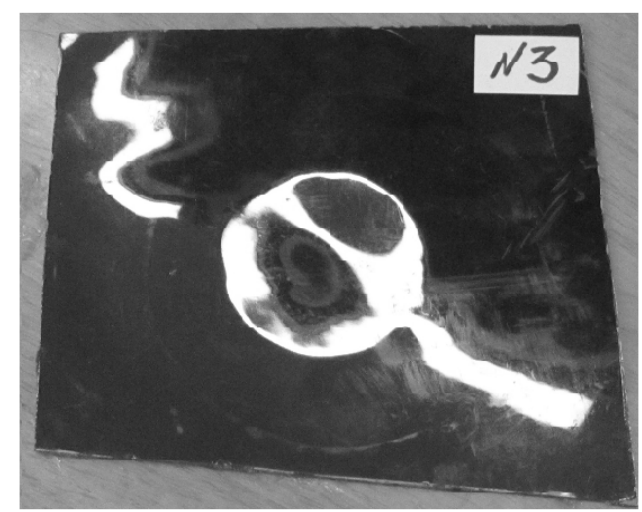

a)
The feature of the power source - MPIS-2 is its work in a serial regime as a generator of a designated quantity repetition of the current pulses which enter to the inductor winding and which excite pulses of the force attraction to the screen plane of the dent metal.

The main data of the MPIS - 2 in experiment are the following.

1. The stored energy is equaled to $\sim 2,4 \mathrm{~kJ}$, the voltage is $\sim 1500 \mathrm{~V}$, the current in pulse is equaled to $\sim 12 \mathrm{KA}$

2. The working frequency in discharge is $\sim 1500 \mathrm{~Hz}$.

3. The frequency repetition of the current pulses is $\sim$ $5 \mathrm{~Hz}$.

4. The quantity of the force attraction pulses what was necessary for the sheet blank flattening is $\sim 18 \div 20$.

The separate plates of the sheet metal body covering automobiles "Subaru" and "Citroen" were taken as the experimental samples. Their dimensions were $\sim 0.15 \mathrm{~m} \times 0.15 \mathrm{~m}$. The thickness of the samples was $~$ $0,0008 \div 0,001 \mathrm{~m}$. The depth of the dents which were created artificially was $\sim 0,0018 \div 0,002 \mathrm{~m}$. Their external diameter was $\sim 0,05 \mathrm{~m}$.

Besides the separate sheet samples an object of the experiment was a door of the automobile "Audi" represented on Fig.2a as well. The thickness of its steel covering was $\sim 0,001 \mathrm{~m}$. Dimensions of a natural dent were $\sim \varnothing 0.06 \mathrm{M} \times 0.02 \mathrm{~m}$ with depth $\sim 0.0015 \mathrm{~m}$.



Fig 2. The equipment for EMF removing the dents in the whole.

\section{Experimental Test and Results}

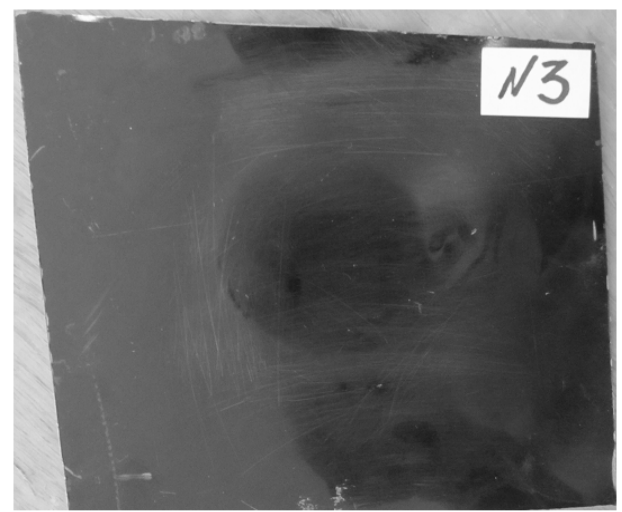

b) 




c)

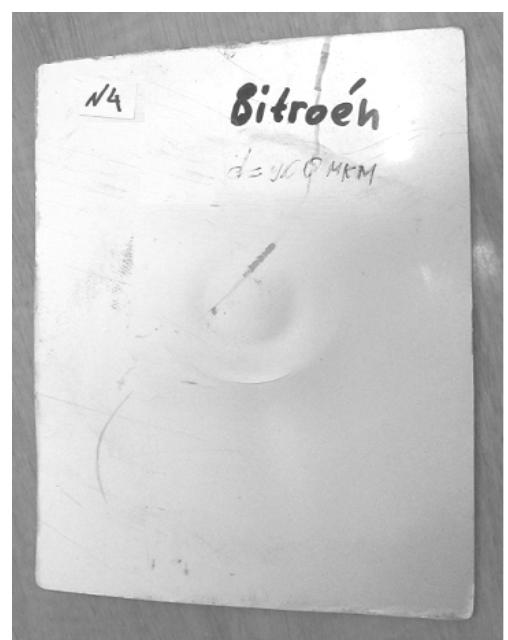

d)

Fig 3. The experimental samples from the different automobile steels, "Subaru" - a) before the force action, b) after attraction; "Citroen" - c) before the force action, d) after attraction.

Preliminary remark: the detailed description of theoretical or methodological underpinnings of the conducted experiments was given in the papers $[6,8]$. Besides it may be found on the Web-Site [10] as well.

After switching on the mains voltage was given on the force step-up transformer primary winding. Charging the capacitors was carried out through the rectifier till a specified level of the stored energy. After this the discharge circuit was automatically closed by a block of the thyristor switches. The discharge current pulses have entered in winding of the exciting inductor (ISAS).

A cycle "charge - discharge" and the force EMF attraction was repeated several times till removing the dent on the object surface of the experimental investigation.

Some results of the external non-contact EMF removing the dents in separate samples of the sheet covering the Japanese and European automobiles are represented on Fig.3.

The first what is necessary to mark as an experimental positive result is a level of the force attraction what provided high effectiveness of the fulfilled production operation. Should remind, a flattening quality was not a problem in this case. The aim of the conducted experiment was only practical test of the ISAS as the tool of the EMF attraction.

The second positive result is saving the protective paintand-lacquer coating. Though a quite important remark has to be done. As it follows from practice saving the paintand-lacquer or other protective coating depends on many factors immediately not connected precisely with EMF attraction of the deformed metal.

Fig.4 illustrates a course of experiment by flattening the steel covering the "Audi" automobile door with natural dent.

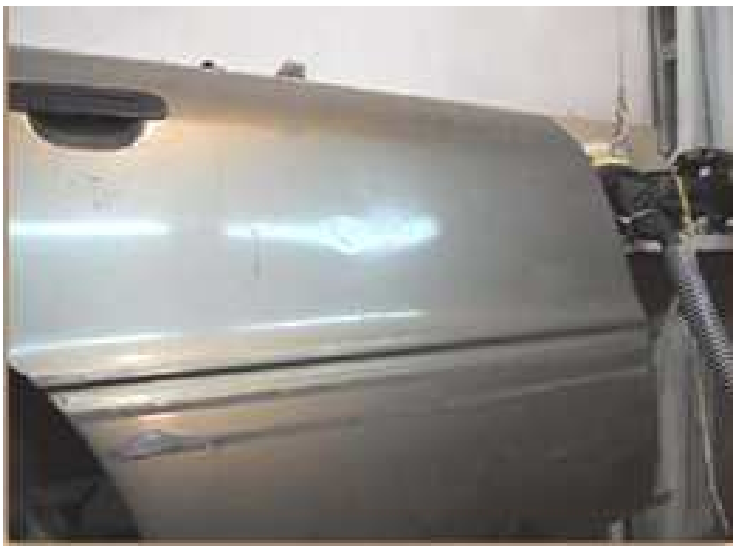

(a)

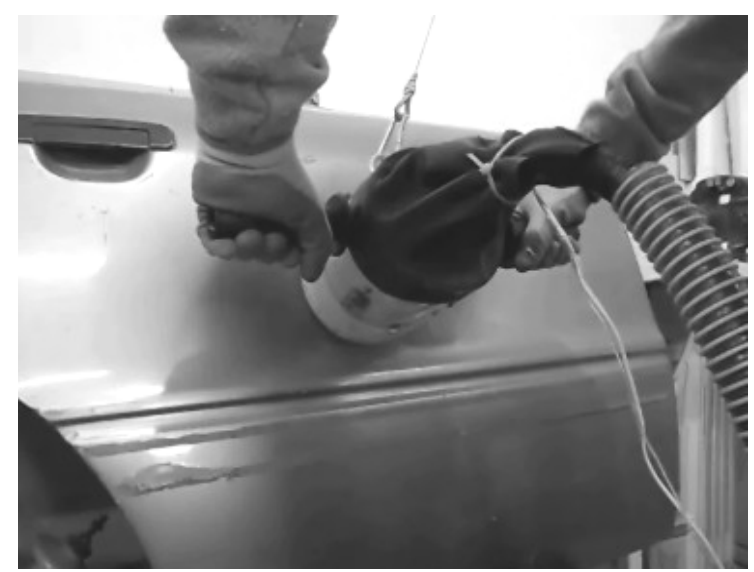

(b) 




(c)

Fig 4. The external EMF flattening in the real repair process, a) the door before flattening; b) the tool ISAS in action; c) the door after flattening.

The experiment by the door restoration of the automobile "Audi" in real condition has demonstrated the effectiveness of fulfilling the manufacturing operation. A visual control of its result has shown the real possibility of the qualitative EMF flattening with help of the suggested tool - "Inductor System with an Attracting Screen (ISAS)".

\section{Conclusions}

1. The "Inductor System with an Attracting Screen (ISAS)" being excited by external source of the magnetic field is successfully tested in the actual repair production operation by the out side noncontact EMF removing the dents.

2. Removing the dents was successfully realized not in idealized conditions for separate steel samples only but in real situation of the element restoration of the automobile body.

3. The got results have demonstrated the high effectiveness of the suggested tool in the principle new advanced repair technology with usage of the pulse magnetic field energy.

\section{Acknowledgement}

I am sincerely grateful to my coworkers of the Laboratory of the Electromagnetic Technologies where the experimental investigations were successfully conducted.
Some of the main results are represented on the Web-site [10].

\section{References}

[1] ELECTROIMPACT is a world leader in design and manufacturing of aerospace tooling and automation. Online available at www.electroimpact.com (shown on 2014).

[2] FLUXTRONIC Specialized in Electromagnetic Dent Removal. Online available at www.fluxtronic.com (shown on 2014).

[3] Welcome to BETAG INNOVATION, efficient repair solutions. Online available at www.betaginnovation.com (shown on 2014).

[4] Meichtry R., Kouba I. 2008. Dent Removing Method and Device. US WO2008/0163661A1.

[5] Yu.V.Batygin, V.I.Lavinsky, L.T.Khimenko, Direction Change of the Force Action upon Conductor under Frequency Variation of the Acting magnetic Field. Proceedings of the 1-st International Conference on High Speed Metal Forming. March 31/April 1, 2004. Dortmund, Germany. P.157-160.

[6] Yuriy V. Batygin, Sergey F. Golovashchen-ko, Andrey V. Gnatov, Pulsed electromagnetic attraction of sheet metals Fundamentals and perspective applications. //Journal of Materials Processing Technology. - Elsevier. - 2013. - № 213 (3). - pp. 444-452.

[7] Batygin, Y.V., Lavinskiy, V.I., Khavin, V.L., 2006. Method of Magnetic Pulsed Treatment of Thin-walled Metal Blanks. Patent of Ukraine 74909.

[8] Yuriy V. Batygin, Sergey F. Golovashchenko, Andrey V. Gnatov, Pulsed electromagnetic attraction of nonmagnetic sheet metals. //Journal of Materials Processing Technology. - Elsevier. - 2014. - № 214 (2). - pp. 390-401.

[9] Yuriy V. Batygin, Andrey V.Gnatov, Evgeniy A. Chaplygin, Irina S. Trunova, Oleg S. Sabokar, 2013. Method of Magnetic Pulsed Attraction of the Metal Billets by SingleTurn Circle Inductor placed over Accessory Attracting Screen. Patent of Ukraine 77579.

[10] Laboratory of the Electromagnetic Technologies. Online available at http://electromagnetic.comoj.com/komerc.html (shown on 2014). 